\title{
El pensamiento computacional en contextos educativos: una aproximación desde la Tecnología Educativa
}

\author{
Computational Thinking in Educational Environments: An Approach from Educational \\ Technology \\ El pensament computacional en contextos educatius: una aproximació des de la \\ Tecnologia Educativa
}

María del Mar Sánchez-Vera ${ }^{\circledR}$

Departamento de Didáctica y Organización Escolar, Universidad de Murcia, Murcia, España

Recibido: 04/07/2019 | Aceptado: 25/10/2019 | Publicado: 20/12/2019

Cómo citar: Sánchez-Vera, M.M. (2019). El pensamiento computacional en contextos educativos: una aproximación desde la Tecnología Educativa. Research in Education and Learning Innovation Archives, 23,24-39. 10.7203/realia.23.15635

Copyright: El/La Autor/a. Open Access: Este es un artículo de acceso abierto distribuido bajo los términos de la licencia Creative Commons AttributionNonCommercial-ShareAlike 4.0 International (CC BY-NC-SA 4.0)

Financiación: Ninguna informada
RESUMEN: El pensamiento computacional ha vivido un resurgir en los últimos años, en los que la comunidad científica y educativa ha puesto su foco de interés sobre él como un aspecto relevante a incorporar de manera curricular. No obstante, la falta de consenso respecto a qué es y cómo trabajarlo tiene como resultado que se aborde de diferentes formas en la educación, lo que repercute directamente en el aprendizaje del alumnado. Desde enfoques más técnicos, que tienen el riesgo de no permitir aprovechar el potencial que tiene de ser integrado en la estrategia didáctica del docente, a enfoques más transversales, que sí profundizan en las posibilidades que tiene en el ámbito de la didáctica, pero que a su vez también incorpora distintas visiones que dificultan su aplicación. Además, se incluyen nuevas perspectivas como el pensamiento computacional "desenchufado". Ante este panorama, se pone en evidencia la necesidad de abordar desde la Pedagogía, y específicamente desde la Tecnología Educativa, el tema del pensamiento computacional, partiendo de las posibilidades que tiene en torno a la alfabetización digital y como medio para expresar ideas con tecnología, y para poder proporcionar propuestas para una mejora real de la práctica en los contextos educativos en relación al uso de la programación y la robótica, partiendo de la premisa de que lo importante no es cómo aprender de robótica, sino cómo aprender con robótica.

PALABRAS CLAVE: pensamiento computacional; programación; robótica; Tecnología Educativa

ABSTRACT: Recently, computational thinking has experienced a resurgence, due to the scientific and educational community has placed its focus of interest on it. However, there is a lack of consensus about what it is and how to work it. That has caused that the approach to computational thinking is addressed in different directions in educational fields. From technical approaches, the potential to be integrated in teachers' teaching strategy could be not considered; from transversal approaches, the possibilities in the field of didactics are included, but they incorporate different visions that makes it application difficult. In addition, new perspectives are incorporated, such as "unplugged" computational thinking. Given this panorama, it is evident the need to address from the pedagogy, and specifically from the Educational Technology field, the topic of computational thinking, starting from the possibilities it has related to digital literacy and as a means to express ideas with technology; to the possibilities to be included in educational contexts around the use of coding and robotics, highlighting that the important aspect is not how to learn robotics, but how to 
learn with robotics.

KEYWORDS: computational thinking; coding; robotics; Educational Technology

RESUM: El pensament computacional ha viscut un ressorgiment en els últims anys, en què la comunitat científica i educativa ha posat el seu focus d'interés sobre ell com un aspecte rellevant a incorporar de manera curricular. No obstant això, la manca de consens respecte a què és i com treballar-té com a resultat que s'aborde de diferents formes en l'educació, el que repercuteix directament en l'aprenentatge de l'alumnat. Des de enfocaments més tècnics, que tenen el risc de no permetre aprofitar el potencial que té de ser integrat en l'estratègia didàctica del docent, a enfocaments més transversals, que sí que aprofundeixen en les possibilitats que té en l'àmbit de la didàctica, però que a la mateixa vegada també incorpora diferents visions que dificulten la seua aplicació. A més, s'inclouen noves perspectives com el pensament computacional "desendollat". Davant d'aquest panorama, es posa en evidència la necessitat d'abordar des de la pedagogia, i específicament des de la Tecnologia Educativa, el tema del pensament computacional, partint de les possibilitats que té al voltant de l'alfabetització digital i com a mitjà per expressar idees amb tecnologia, i per poder proporcionar propostes per a una millora real de la pràctica en els contextos educatius entorn de l'ús de la programació i la robòtica, partint de la premissa que l'important no és com aprendre de robòtica, sinó com aprendre amb robòtica.

PARAULES CLAU: pensament computacional; programació; robòtica; Tecnologia Educativa

\section{Notas de aplicación práctica}

\section{Qué se sabe sobre este tema}

- A principios de este siglo se desarrollaron nuevas conceptualizaciones sobre lo que es y lo que implica el pensamiento computacional. Este hecho, unido al desarrollo de sistemas operativos más intuitivos y la creación de nuevos modelos de robots para las aulas, ha hecho que sea foco de interés actual de la comunidad científica y educativa. De hecho, codificar (coding) es considerada una competencia básica del siglo XXI. No obstante, no existe consenso respecto a su conceptualización y a cómo tendría que integrarse en el currículo y trabajarse en el aula, lo que nos lleva a encontrar una gran diversidad de experiencias y enfoques en torno a la temática.

\section{Qué aporta este trabajo}

- Este trabajo explica y clasifica las diferentes visiones de la comunidad científica y educativa sobre cómo hay que incorporar el pensamiento computacional en las aulas, tratando de analizar si la incorporación de la robótica y la programación en el currículo actual está promoviendo realmente el pensamiento computacional en la educación.

- El análisis que se realiza del pensamiento computacional se aborda desde los principios básicos de la Tecnología Educativa, rescatando sus bases y las contribuciones que hace en el ámbito de la Didáctica.

\section{Implicaciones para la práctica y/o política}

- El análisis que se presenta aborda el pensamiento computacional desde la alfabetización mediática, la competencia digital y el estudio de los medios. Las conclusiones del trabajo pueden ayudar, por un lado, a plantearnos la incorporación del pensamiento computacional en el currículo de manera transversal, de tal manera que éste nos permita desarrollar las habilidades necesarias para poder crear y expresarnos mediante la tecnología. Y por otro lado, puede servir para plantearse el tipo de formación que deben recibir los docentes para hacer una buena integración del pensamiento computacional en el aula.

\section{INTRODUCCIÓN}

En pleno auge de la programación y de las primeras experiencias con robótica en el ámbito científico, en los años 60-70, Papert es precursor en la introducción de la 
programación y la robótica en las escuelas de la época, gracias a las propuestas del lenguaje de programación LOGO y el robot Turtle para la enseñanza de la programación a niños en los colegios americanos. La incorporación de la programación a la enseñanza se consideró relevante hasta tal punto que se argumentaba la necesidad de que los estudiantes universitarios de todas las disciplinas aprendieran a programar (Grover y Pea, 2013; Pérez-Narváez y Roig-Vila, 2015). Durante esta época se comienza a considerar también la importancia de las conexiones entre la programación y los estilos de pensamiento que desarrollamos al trabajar con la tecnología, como antecedente a lo que actualmente denominamos pensamiento computacional.

De hecho, Papert se preocupó no solo de desarrollar tecnología, sino de analizar cómo los seres humanos interaccionábamos con ella. Influenciado por Piaget, al que conoció en Ginebra, enarboló su propia teoría del aprendizaje con robótica, a la que llamó "construccionismo". Lo interesante de la propuesta de Papert es que no centra la importancia en la "máquina", sino en el tipo de pensamiento que permite desarrollar y la manera de trabajar con ella. Papert fue pionero en cuanto a considerar que los alumnos podían programar y utilizar robots como medio de aprendizaje en la escuela.

No obstante, a pesar de todas las posibilidades que generó la visión de Papert, el desarrollo de sistemas operativos más intuitivos durante las siguientes décadas hizo que se pudiera utilizar la tecnología de manera sencilla sin tener que aprender a programar. A esto se unía el hecho de que los lenguajes de programación eran complicados de manejar y que los docentes no supieron adaptar didácticamente las propuestas con el robot. Estas afirmaciones han sido realizadas por discípulos del trabajo de Papert, que posteriormente recuperaron los postulados de su mentor, para seguir trabajando sobre pensamiento computacional (en adelante PC) desde perspectivas más amplias.

El resurgir del PC se considera que se articula en torno a la figura de Wing, que en 2006 utilizó este concepto en un artículo, para referirse al PC como el proceso de pensamiento que permite resolver problemas, diseñar sistemas y entender los mecanismos del comportamiento humano, utilizando los conceptos y procedimientos de la tecnología.

A nivel técnico han surgido, además, nuevos lenguajes de programación, que cada vez se han hecho más intuitivos y adaptados a los niños, usando elementos visuales como los bloques de colores, y definidos como un "entornos de programación mediados simbólicamente” (Pérez-Narváez y Roig-Vila 2015, p. 7).

Lo que está claro es que, el resurgir a nivel científico, unido al desarrollo exponencial de la robótica y el software relacionado con la programación para el alumnado hace que el PC esté en el foco de interés de la comunidad científica y educativa. Lo evidencia el interés de las distintas instituciones en promover este tipo de actividades. Codificar (coding), de hecho, ha sido incluido específicamente como una de las competencias básicas del siglo XXI (Bocconi, Chioccariello, Dettori, Ferrari, y Engelhardt, 2016) y se promueve en la Unión Europea a partir de experiencias como la Codeweek.

Debido a todo esto, existe un interés creciente en la comunidad educativa en relación a la introducción del pensamiento computacional en las aulas. Esta afirmación se puede comprobar en los datos obtenidos por la encuesta del INTEF al profesorado (MEFP, INTEF, y CNIIE, 2018), que indica que la mayor parte del profesorado encuestado estaba de acuerdo en incorporar actividades en el aula para trabajar la robótica, la programación y el PC.

Existen tres conceptos básicos que se mencionan cuando se aborda la temática: programar, robótica y PC, que se utilizan conjuntamente en muchas de las propuestas educativas, pero resulta imprescindible diferenciar y delimitarlos antes de avanzar, para posteriormente poder comprender cómo interaccionan unos con otros. Una conceptualización interesante es la propuesta por Reina (2018, p. 4), que los define de este modo: la robótica consiste en la "construcción de un mecanismo o robot que se programa a través de un software [...]; el lenguaje de programación es lo que nos 
permite comunicarnos con el robot o dispositivo digital y el PC es el proceso cognitivo implícito al programar que permite resolver problemas a partir de una secuencia de pasos ordenada".

La robótica educativa supondría, por tanto, el desarrollo de propuestas educativas que incorporen el uso de robots en las aulas. A este respecto vamos a encontrar diversas perspectivas sobre su integración en entornos educativos: una se refiere a la integración mediante actividades independientes y específicas (o actividades extracurriculares); y otra a la integración de manera transversal e interdisciplinar, desarrollando diferentes aspectos curriculares.

Podemos preguntarnos si es posible desarrollar el PC sin programación y sin robótica. Al menos desde las perspectivas conceptuales que manejan muchos de los autores actuales, como Bers (2018), Grover y Pea (2013) y Wing (2006), sí podemos hacerlo. Este enfoque se adoptó a principios de este siglo, cuando comenzó a hablarse de la importancia del PC como una nueva alfabetización que proporciona las habilidades necesarias para resolver problemas en diferentes contextos. Es lo que se llama PC "desenchufado" y abre una nueva perspectiva: es posible desarrollar el PC sin programar. No obstante, es cierto que, aunque se indica que no es necesario programar ordenadores para desarrollar el PC, hacerlo ayuda a ponerlo en práctica (Adell, LlopisNebot, Esteve, y Valdeolivas, 2019).

Por lo tanto, la robótica y la programación son elementos que están relacionados con el PC, y programar y usar robots ayuda a desarrollarlo, aunque podamos también trabajarlo sin programación y sin robótica.

Ante este panorama, ¿estamos repitiendo errores del pasado al incorporar los recursos sin tener en cuenta el papel del docente y los principios de integración de los medios? Este artículo pretende analizar la incorporación del pensamiento computacional en la educación, realizando una aproximación desde la Tecnología Educativa.

\section{VISIONES DE LA COMUNIDAD CIENTÍFICA SOBRE EL PENSAMIENTO COMPUTACIONAL}

Para Piaget y Vigotsky, el pensamiento es el elemento fundamental del aprendizaje. "El pensamiento funciona cuando forma conceptos en el cerebro, resuelve problemas y toma decisiones, todo esto se consigue cuando está un medio para expresar ideas, así como para concebir las categorías y los conceptos de pensamiento" (Jaramillo y Puga, 2016, p.35). Específicamente, el PC es un tipo de pensamiento analítico. Comparte con el pensamiento matemático la manera de abordar la solución a un problema, con la ingeniería comparte la manera en la que se aborda el diseño y la evaluación de un sistema complejo que opera en el mundo real. Y con el pensamiento científico la forma en la que abordamos la inteligencia, la mente y el comportamiento humano (Wing, 2008).

A nivel psicológico, Jaramillo y Puga (2016) indican que Papert planteaba la vinculación del PC a través de la robótica con el constructivismo, por el hecho de que el PC requiere, no solo la adquisición de conceptos, sino la materialización de estos. El mismo Papert (1980) y las personas que han trabajado con él (Bers, 2010, 2018), han tratado de seguir esta corriente "constructivista" influenciada por Piaget. Aunque más que constructivista, Bers (2018) la ha denominado como "construccionistas", siguiendo la estela de Papert y tratando de justificarlo en torno a los procesos de aprendizaje que se ponen en marcha, relacionados con la construcción y experimentación con robots.

Es cierto que programar y usar robots no implica que automáticamente produzcamos PC, pero Papert ya mencionaba en 1980 que cuando trabajamos con robots contribuimos a desarrollarlos, porque lo que estamos haciendo es aprender una nueva manera de comunicarnos con tecnología. El autor indica, incluso, que es una manera de "hablar matemáticas". Un adulto que sepa "hablar matemáticas" no necesita resolver 
complejas ecuaciones, sino haber desarrollado los mecanismos de razonamiento básico de las matemáticas que le permita enfrentarse a situaciones complejas que requieran del razonamiento matemático para resolverlas. Por ejemplo, "cuando le pedimos a un robot que haga un giro circular, la programación no le da una respuesta directa, sino que anima a los alumnos a encontrar su propia solución, que puede ser yendo hacia delante y hacia atrás, girando en un sentido u otro, etc." (Papert , 1980, p. 206). Esto puede ayudar a desarrollar procesos mentales relacionados con la resolución de problemas (Furber, 2012). De hecho, Papert (1980) indica que para desarrollar su propuesta educativa con robótica tuvo en cuenta la manera en la que un niño aprende a hablar y desarrollar su lenguaje.

Blikstein (2013) destaca la importancia de Papert como pionero de la integración de las tecnologías en la educación y su visión acerca de que estas no son solo herramientas que optimizan los procesos de enseñanza-aprendizaje, sino recursos que tienen un poder emancipatorio en las manos de los niños, porque les permite crear, expresar y compartir ideas. En un entorno de aprendizaje "construccionista" no hay un currículum cerrado, los niños usan la tecnología para crear proyectos, y los profesores son facilitadores del proceso (Blikstein, 2013). De hecho, para Papert, el robot es un objeto con el que pensar, y la programación un lenguaje con el que podemos expresarnos.

Otra conceptualización sobre el PC, que está bastante extendida es la que aporta la Sociedad Internacional para las Tecnologías en Educación (ISTE por su sigla en inglés) y la Asociación de Maestros de Ciencia de la Computación (CSTA por su sigla en inglés), que elaboran una definición operativa de PC y lo definen como "un proceso de solución de problemas que incluye (pero no se limita a) las siguientes características (ISTE y CSTA, 2011, p. 26)":

- Formular problemas de manera que permitan usar ordenadores y otras herramientas tecnológicas para solucionarlos.

- Organizar datos de manera lógica y analizarlos.

- Representar datos mediante abstracciones, como modelos y simulaciones.

- Automatizar soluciones mediante pensamiento algorítmico (una serie de pasos ordenados.

- Identificar, analizar e implementar posibles soluciones con el objeto de encontrar la combinación de pasos y recursos más eficiente y efectiva

- Generalizar y transferir ese proceso de solución de problemas a una gran diversidad de contextos.

No obstante, diversos autores confirman que no existe acuerdo en torno a la conceptualización del PC (Adell, Esteve, Llopis, y Valdeolivas, 2017; González, Estebanell, y Peracaula, 2018; Grover y Pea, 2013; Lye y Koh, 2014) y esto se traslada al hecho de que ha sido introducido de manera muy diferente en las escuelas a nivel curricular. Incluso la propia denominación de "computacional" es debatida por expertos en el tema, al hacerlo parecer algo meramente técnico (Bocconi et al., 2016). A partir de las revisiones y el trabajo realizado por varios autores, se aporta un análisis en torno a las diferencias encontradas en relación al PC.

A nivel general, se pueden identificar dos corrientes principales referidas a la concepción de lo que es y lo que implica el PCen los últimos años. Una es la que representa Wing $(2006$; 2008; 2011) y otra es la que promueve Bers (2010; 2018).

La definición de PC de Wing, que aborda en 2006 y reforma en 2008, implica que el PC es un tipo específico de pensamiento que permite la resolución de problemas e implica desarrollar capacidades distintas, por ejemplo, ser capaz de diseñar soluciones para ser ejecutadas por un ordenador, un humano, o una combinación de ambos (Adell 
Tabla 1. Diferencias entorno a la definición y conceptualización del pensamiento computacional (a partir de Bocconi et al., 2016; Grover y Pea , 2013; Lye y Koh, 2014)

El tipo de conceptualización

La finalidad

La relación con la programación

Dónde aplicarlo a nivel curricular

En relación a cómo se introduce en las escuelas
Informático

Psicológico

Educativo

Multidisciplinar

Autores que se suscriben a la definición de Wing, relacionada con la resolución de problemas

Autores que tratan de diferenciar el pensamiento computacional de otras formas de pensar

Autores que lo incorporan dentro de la competencia digital o la alfabetización digital

Autores que lo caracterizan de manera abstracta sin llegar a definirlo. Por ejemplo: "la capacidad de descubrir"

Programar, utilizar, desarrollar tecnologías

Desarrollar competencias para resolver problemas

Implica ambas cosas

Adquirir el pensamiento computacional no requiere codificar o programar, porque el pensamiento computacional es una manera de resolución de problemas

Programar contribuye y ayuda a desarrollar el pensamiento computacional (para algunos autores es imprescindible)

Codificar y/o programar es uno de los elementos básicos del pensamiento computacional y son necesarios para desarrollarlo

En una asignatura específica de robótica o de informática

Como tema o parte de asignaturas de ciencias experimentales (STEM)

Transversalmente en todas las áreas/disciplinas

Cambia en función del nivel educativo, con desarrollo transversal en Infantil y Primaria y tiende a ser una asignatura especifica en Secundaria

Empatizando en el desarrollo de sus componentes (como la abstracción)

Apoyando y/o promoviendo el pensamiento computacional en otras disciplinas

Desarrollando actividades específicas para alumnado

et al., 2017, p.8).

Bers (2018) indica que esta definición de Wing es limitada, porque, aunque destaca la importancia de la resolución de problemas en el marco del PC, la perspectiva de Wing (2006, 2008), según Bers (2018), esconde una de las principales potencialidades del PC que es la posibilidad de expresar ideas y de crear, como lo hacemos cuando programamos. Bers (2018) se fundamenta en los trabajos de Papert para indicar que la noción de expresión es relevante en el concepto de PC. Esta autora defiende que el PC es una nueva forma de alfabetización, ya que la programación, al igual que la escritura, es un medio para expresarnos. Al igual que el lenguaje puede tener múltiples representaciones y resultados, los lenguajes de programación nos permiten expresarnos de maneras diferentes y crear distintos productos. Bers (2018) indica que, en definitiva, la resolución de problemas es una manera de expresar una solución a un problema, por lo que estaría enmarcada en una meta más global que es la de la creación, 
por lo tanto, la valía del PC está en la posibilidad de expresar y el desarrollar una idea. Del mismo modo que el lápiz es la herramienta que permite escribir, el ordenador es la herramienta que permite crear. No promueve Bers que todos seamos programadores o informáticos, sino que tengamos las habilidades necesarias para poder crear y expresarnos mediante la tecnología, como parte de la alfabetización necesaria en el mundo actual.

\section{COMPONENTES DEL PENSAMIENTO COMPUTACIONAL}

Si en torno a la definición de PC existe controversia, también la hay cuando tratamos de conocer los componentes que lo configuran, ya que estos elementos varían en función de la perspectiva que se adopte. No obstante, existen una serie de componentes comunes en la clasificación que se hace desde la literatura científica que podemos destacar.

Entre estos elementos comunes se encuentra la abstracción. Wing (2008) indica que la base del PC es la abstracción. Las abstracciones en informática son simbólicas. Específicamente, un lenguaje de programación es una abstracción de un conjunto de cadenas, cada una de las cuales, cuando se interpreta, tiene un efecto de cálculo. El algoritmo es lo que nos permite seguir las instrucciones para operar una actividad.

El pensamiento abstracto es un proceso mental en el cual se destaca lo principal de una determinada acción (Jaramillo y Puga, 2016, p. 41). Podríamos decir que la abstracción trata de reducir la complejidad para poder identificar los elementos clave que nos permitan destacar lo que es más relevante. Piaget y Vygotsky ya analizaron la importancia del pensamiento abstracto en el desarrollo del aprendizaje de los niños.

Como se ha mencionado, son varios los autores que realizan clasificaciones de los componentes del PC (Bers, 2018; Brennan y Resnick, 2012; Zapata-Ros, 2015), pero existen una serie de conceptos computacionales que son relativamente comunes, como el de secuencia, ciclo, eventos, paralelismo y condicionales, que formarían parte del PC (Brennan y Resnick, 2012; Lye y Koh, 2014). Específicamente, el concepto de secuencia es bastante común en todas las aproximaciones que se realizan. La secuencia es la que nos permite desarrollar una manera específica de resolver los problemas.

Catlin y Woollard (2014) también tratan de realizar una conceptualización del PC, tratando de describir los subconceptos que lo compondrían y el desarrollo de las competencias que supondrían el trabajarlos adecuadamente.

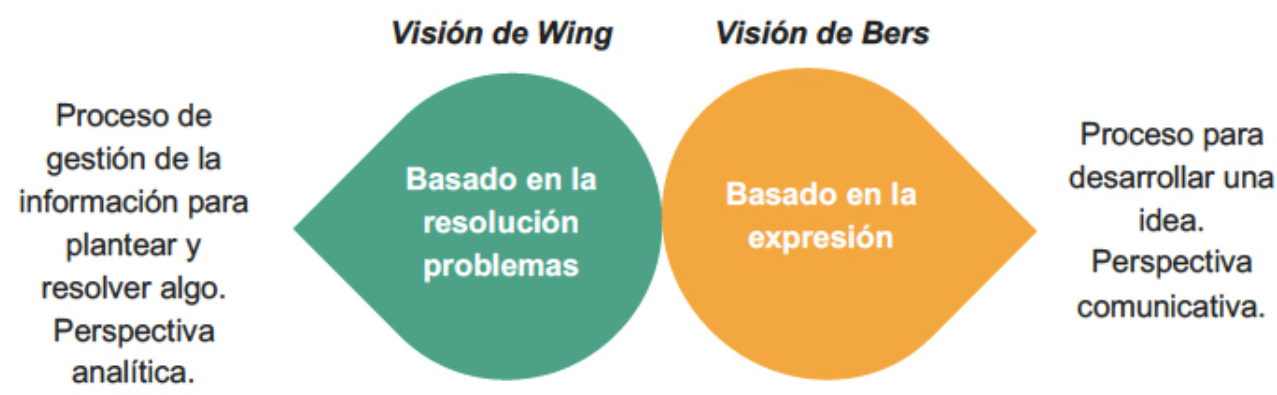

Figura 1. Diferencias en las visiones de Jeanette Wing y Marina Bers en torno al concepto de pensamiento computacional 
Tabla 2. Pensamiento computacional: conceptos y competencias (Catlin y Woollard, 2014, p. 3)

\begin{tabular}{ll}
\hline Conceptos & Competencias \\
\hline Abstracción & $\begin{array}{l}\text { Enfrentarse a un problema a partir de la reducción de detalles } \\
\text { innecesarios }\end{array}$ \\
Algoritmo & Identificar un proceso y una secuencia de eventos \\
Descomponer & Dividir o descomponer artefactos, procesos o sistemas en partes \\
Generalizar & Identificar los patrones y las características comunes entre \\
& artefactos, procesos o sistemas \\
Análisis lógico & Aplicar e interpretar la lógica booleana (estructura que \\
& esquematiza las operaciones lógicas) \\
Evaluación & Sistematizar (a través de criterios) y hacer un juicio de valor \\
\hline
\end{tabular}

\section{LA INCORPORACIÓN DE LA ROBÓTICA Y LA PROGRAMACIÓN EN EL CURRICULUM ¿ESTAMOS DESARROLLANDO EL PENSAMIENTO COMPUTACIONAL?}

En muchos países se está integrando la robótica y la programación como parte del currículo educativo. A nivel europeo, desde 2014 se promueve que los distintos países incorporen la programación informática en las escuelas para ayudar a solucionar el desempleo juvenil (MEFP et al., 2018). La European Schoolnet (Balanskat, Engelhardt, y European Schoolnet, 2015) realiza una aproximación al estado de la enseñanza con robótica y la programación en los sistemas educativos europeos. Encuentra que existen diversas maneras de integrar esta temática en la educación: como una asignatura específica y diferente (robótica, programación, etc.), en el marco de una asignatura de TIC, o como tema o parte de otra asignatura curricular (normalmente informática o matemáticas). El informe indica, que 12 países habían establecido una asignatura específica de programación, mientras que otros 13 la incorporaban en una asignatura de TIC y 7 en el marco de otras asignaturas (principalmente matemáticas). Aunque estas políticas difieren mucho dentro del mismo país en las distintas regiones, debido a la delegación de competencias en materia de educación y las políticas regionales.

En nuestro país, la ley educativa actual promueve el uso de las TIC de manera general como elemento transformador del sistema educativo. Sin embargo, son las Comunidades Autónomas las que, hasta ahora, han especificando la concreción curricular que favorece la incorporación en el aula de la programación, la robótica y el PC.

En la figura anterior aparecen en color azul aquellas que lo han hecho en Primaria; verde, en Secundaria; y en morado, en ambos niveles educativos. Las Comunidades Autónomas que no participaron en el estudio aparecen en gris (MEFP et al., 2018, p.28).

Hemos visto cómo encontramos dos visiones acerca de cómo incorporar el PC a través de la programación y la robótica en las aulas. Estas dos visiones son las que denomina el INTEF (MEFP et al., 2018) como enfoque específico, que se refiere a introducirlo como una asignatura específica y centrándose en la enseñanza de la tecnología; y un enfoque transversal, focalizado en la incorporación en otros contextos educativos o asignaturas no relacionadas con la tecnología. La primera asume que la mera incorporación de estos recursos ya posibilitará el desarrollo del PC, mientras que otra entiende la importancia de la planificación educativa y la estrategia didáctica del docente, e incluso valora la posibilidad del desarrollo del PC sin tecnología. Resulta interesante ver que, conforme se va avanzando en el sistema educativo, se percibe cada vez menos el potencial de la transversalidad del PC.

Resultan interesantes también los datos presentados por Lye y Koh (2014), que realizaron un estudio en el que analizaron 27 artículos sobre programación en la educación. Encontraron que uno de los grandes usos que se hacía de la programación era la 


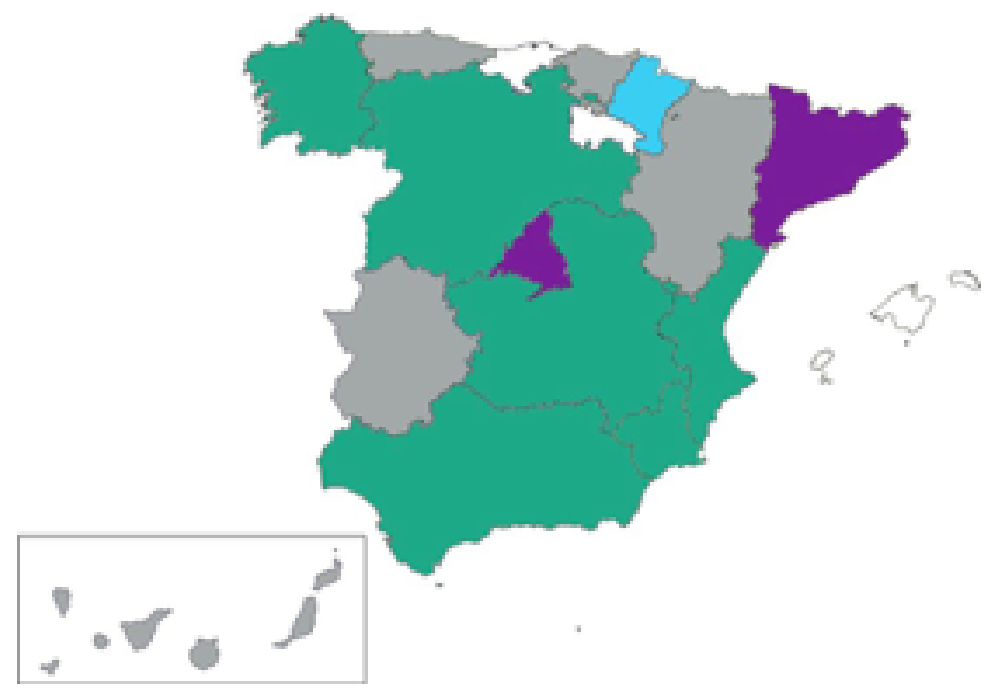

Figura 2. Comunidades Autónomas que han incluido nuevas asignaturas o contenidos sobre programación, robótica y PC (MEFP et al., 2018)

creación de historias digitales y juegos. Los resultados encontrados son positivos y son considerados por los autores tareas interesantes para trabajar en la educación porque suponen la "creación" de algo, la puesta en práctica de una idea, sin embargo, cuando incorporamos la programación y la robótica solo de modo anecdótico o extracurricular, parece que aún queda un amplio abanico de posibilidades que no se terminan de aprovechar, si queremos trabajar el PC y los procesos cognitivos que implica.

Tratando de responder a la pregunta planteada en este apartado, podríamos indicar lo siguiente (Bers, 2018; González et al., 2018; MEFP et al., 2018; Reina, 2018):

- La incorporación de la programación y la robótica ayuda al desarrollo del PC, pero no lo asegura.

- El PC puede trabajarse con programación y robótica o sin programación y robótica como el PC desenchufado.

- El trabajo con programación y robótica desde perspectivas extracurriculares y excesivamente técnicas hace que se desaprovechen las potencialidades de los recursos para ser incorporados en contextos educativos.

- A nivel curricular, los esfuerzos para incorporar la programación, la robótica y el PC se han orientado principalmente a Educación Secundaria (y en algunos casos también en Educación Primaria), aunque existe una tendencia actual a valorar también la importancia de trabajarlo desde la Educación Infantil.

- El PC puede integrarse de manera transversal, y la programación y la robótica son recursos que podemos utilizar en el marco de distintas áreas de conocimiento. Es importante rescatar los planteamientos tradicionales de la Tecnología Educativa en cuanto a la integración de los recursos en la educación.

- El papel del docente es clave para la integración de estos recursos de manera adecuada en contextos educativos.

\section{POTENCIALIDADES Y DIFICULTADES DE LA INTEGRACIÓN DEL PENSAMIENTO COMPUTACIONAL EN LA EDUCACIÓN}

Como hemos visto, el desarrollo del PC podría ayudar a favorecer habilidades para el desarrollo de la lógica y el pensamiento matemático, favoreciendo la capacidad de 
razonar del alumnado. El potencial de la transversalidad de este tipo de pensamiento puede favorecer la interacción de diferentes áreas de conocimiento y ayudar a desarrollar un aprendizaje más globalizado.

Los motivos para incorporar el PC pueden ser varios:

- Permite utilizar programación, videojuegos, robots y elementos que pueden despertar la curiosidad y la motivación del alumnado Bers (2018), así como ser un medio con el que tratar de despertar conciencias científicas y promover el estudio de las STEM.

- Permite el desarrollo de tareas creativas, utilizando o no, tecnologías (García, Deco, y Collazos, 2017).

- Permite desarrollar la habilidad para pensar de manera sistemática y la mejora en el campo de las ciencias experimentales (Lye y Koh, 2014) y ayudar a los alumnos a pensar de manera diferente, solucionar problemas y analizar el entorno con una perspectiva distinta (Bocconi et al., 2016).

- Puede servir al estudiante para redefinir su relación con el resto de las asignaturas y replantearse el proceso de aprendizaje (González et al., 2018). En este sentido, puede enmarcarse en distintas estrategias metodológicas docentes, y el papel del docente se hace fundamental al convertirse en un facilitador o guía del proceso.

- Supone el desarrollo de una nueva alfabetización, necesaria en el mundo en el que vivimos, que nos ayuda a no ser sólo consumidores digitales, sino creadores y participantes activos con las tecnologías (Bers, 2018; Zapata-Ros, 2015).

- Puede ayudar a la mejora y crecimiento de un país, contribuyendo a capacitar en el uso de tecnologías a personas que pueden solventar determinadas necesidades laborales (Bocconi et al., 2016). Aunque esta argumentación puede ser cuestionada si se establece como única motivación para la incorporación de este en la escuela, por la visión neoliberal de la educación, que la reduce a la mera formación de trabajadores (Adell et al., 2019).

En cuanto las dificultades en torno al PC, la mayoría de las consideraciones que se incluyen a continuación no son críticas al PC en sí mismo, sino hacia cómo se está aplicando en contextos escolares, o en relación al desarrollo de enfoques reduccionistas o solamente técnicos que no aprovechan las potenciales que podría tener trabajar el PC en las aulas.

- Entender qué es el PC y lo que implica. Que en el ámbito científico los expertos no se pongan de acuerdo sobre lo qué es el PC y lo que implica, conlleva que el entendimiento del mismo y las reflexiones acerca de cómo llevarlo a la práctica se compliquen. Esto lleva a considerar en ocasiones que con aplicar la robótica y la programación se desarrolla el PC. Como indican González et al. (2018), aunque programar y usar robótica ayuda a adquirir el PC, es necesario de acciones específicas y del diseño de tareas educativas bien integradas en la docencia para poder lograr trabajarlo adecuadamente.

- Mala aplicación curricular. Un enfoque técnico y reduccionista tiende a considerar que poner a los niños a programar líneas de código permite desarrollar el PC. Sin embargo, programar sin sentido y sin una finalidad conectada con el currículo quedará como una actividad aislada y sin fundamento. Algunos autores (Valverde, Fernández, y Garrido, 2015; Zapata-Ros, 2015) consideran que este modelo educativo está siendo aplicado en muchas decisiones políticas que tratan de incorporar erróneamente la programación en las aulas desde un enfoque excesivamente técnico. Recordemos que el PC permitiría el desarrollo de procesos cognitivos 
superiores, y el desarrollo de tareas de este tipo no es sencillo. El trabajo del profesorado en el diseño de las tareas se hace, por lo tanto, fundamental.

- Dar valía únicamente a la práctica. Cuando hablamos de programar o construir robots estamos hablando de tareas prácticas de gran utilidad, sin embargo, aunque una de las potencialidades del PC es la creación, todo el proceso de trabajo para llevar a cabo la tarea es también importante en sí mismo Bers (2018). La práctica es importante, pero el proceso de trabajo, y los principios conceptuales que nos llevan a realizar la tarea, también lo son en el marco del PC. No obstante, algunas prácticas con programación y robótica se centran únicamente en el diseño de un producto vistoso, dejando a un lado el resto del proceso de trabajo.

- Poca investigación y desarrollo desde la Pedagogía. No existen todavía investigaciones que hagan un buen análisis de la implementación del PC en las aulas de diferentes niveles educativos y que traten de conocer si se han desarrollado o no todas esas posibilidades que parece tener (Adell et al., 2019; González et al., 2018). Además, cuando analizamos los referentes en el PC, como es relativamente entendible, su mayoría provienen del mundo de las matemáticas, la informática y la ingeniería. De hecho, algunas de las propuestas curriculares acerca de cómo incorporarlos en las aulas proviene también de profesionales en el ámbito de STEM. No es que esto sea un factor negativo en sí mismo, ya que muchas de las aportaciones, como las de Bers $(2008,2010,2012,2018)$ son sumamente interesantes, pero sí pone en evidencia la necesidad de abordar desde la Pedagogía, y desde el ámbito de la Tecnología Educativa específicamente, el tema del PC, para contribuir al desarrollo científico en torno al tema y proporcionar una mejora real desde la práctica en los contextos educativos. Ya que la postura que se está planteando es, por ejemplo, en relación a la robótica, que lo importante no es cómo aprender de robótica, sino como aprender con robótica.

\section{CONCLUSIONES}

La Tecnología Educativa, como disciplina situada en el ámbito de la Didáctica (Bautista y Alba, 1997), tiene la peculiaridad de, entre otras cosas, enseñar cómo podemos integrar los medios en los procesos de comunicación educativos, con la idea de mejorar los procesos de enseñanza-aprendizaje, y todo esto ocurre a su vez dentro de un proceso de enseñanza-aprendizaje (Martínez , 2016, p.19). Por lo tanto, la Tecnología Educativa no debería referirse solamente a productos finales, sino prestar atención a los procesos de aprendizaje que se producen, por lo que se implica un juicio crítico en torno a las TIC (Cabero, 2003). Sería un error entender la tecnología como un producto y no como un proceso (Amiel y Reeves, 2008), sin embargo, resulta curioso como durante estos últimos años se han repetido algunos de los errores que ya se produjeron con la incorporación de los primeros medios en la enseñanza, ya que se ha dado por supuesto con demasiada ligereza que el simple hecho de introducir estas tecnologías en los centros y las correspondientes acciones formativas técnicas sobre el uso de los medios era garantía de poder ofrecer una enseñanza de calidad y una enseñanza innovadora (Prendes, 2004). Esto mismo puede ocurrir con el PC, si asumimos que la mera incorporación de la robótica y la programación desarrollarán el mismo. En realidad, serían necesarias toda una serie de estrategias didácticas para que el diseño, selección e implementación de estos recursos sea la adecuada.

Además, el impacto socio-cultural de las tecnologías nos hace ser críticos desde la Tecnología Educativa con las nuevas tendencias o modas en relación al uso de determinadas herramientas en la educación, porque tras la perspectiva de enriquecer o mejorar la educación, puede esconderse otro tipo de intencionalidades políticas y/o empresariales. En cuestiones referidas al PC sería importante, por tanto, buscar la 
síntesis entre la acción empresarial (realizaciones tecnológicas) y la acción académica (reflexiones), estableciendo equipos multidisciplinares, ya que la Tecnología Educativa debe ser un cauce para la creatividad, sin dejar de ser críticos con los medios y sus repercusiones sociales (García-Valcárcel, 2016).

Ante este panorama, la Tecnología Educativa nos puede ayudar a tener en cuenta cómo abordar el PC en educación desde diferentes perspectivas:

\section{- La alfabetización y competencia digital:}

Si partimos de la perspectiva que anteriormente hemos detallado de Bers (2018), trabajar el PC podría suponer el desarrollo de una nueva alfabetización. Cabe preguntarse entonces la relación que puede existir del mismo con la alfabetización y/o la competencia digital. Podemos entender la alfabetización digital como "un conjunto de habilidades esenciales para la vida en la mayoría de los casos y como un talante especial para afrontar problemas científicos y tecnológicos" (Zapata-Ros, 2015, p.11). En este sentido Papert ya hablaba en 1980 de la importancia de entender que programar es aprender a "hablar" en un lenguaje específico, el lenguaje que hablan los ordenadores.

Este autor desarrolla en su libro el concepto de "fluidez tecnológica" (Technological fluency) para contrastar el de "alfabetización tecnológica" (Technological literacy). Define "fluidez tecnológica" como la habilidad de utilizar y aplicar las tecnologías sin esfuerzo, tal y como una persona desarrolla un uso propio de su lengua (Bers, 2010).

Bers (2018) indica que la alfabetización relacionada con saber leer y escribir ha permitido a la humanidad representar sus ideas en textos, textos con distinta finalidad (un poema, una carta, un cartel publicitario), pero productos, en definitiva, que nos permiten comunicarnos. Cuando una persona programa puede crear, y, por lo tanto, puede expresar sus ideas, ya sea a través de un videojuego o un robot, por ejemplo.

Adell et al. (2019) destacan la complicada relación del PC con la competencia digital, ya que, en realidad, este concepto presenta elementos comunes no sólo con el de competencia digital, sino también con el de competencia mediática, o competencia informacional. De hecho, la programación aparece en el marco de la competencia digital de la Unión Europea (DigComp 2.1).

No obstante, si se pretende abordar el PC desde el desarrollo de la competencia digital, deberíamos tener en cuenta las perspectivas actuales en relación este concepto que se trabaja desde la Tecnología Educativa desde hace años (Carrera y Coiduras, 2012; Durán-Cuartero, Prendes, y Gutiérrez-Porlán, 2019; Gisbert-Cervera, Espuny-Vidal, y González-Martínez, 2011; INTEF, 2017). Desde esta perspectiva, la competencia digital "no es solamente entender cómo usar las tecnologías, sino que indefectiblemente pasa por la necesidad de comprender el profundo impacto de las mismas en un mundo digital y promover la colaboración para integrarlas de modo efectivo" (Durán-Cuartero et al., 2019). Por tanto, aprender a programar no sería únicamente una habilidad técnica, sino la alfabetización del siglo XXI, que permite no sólo ser consumidores, sino también productores digitales.

- El estudio de los medios:

Hemos visto que el PC se puede trabajar de diferentes maneras, "desenchufado" (planteando el diseño de actividades sin tecnología, a veces incorporando juegos o materiales que ayudan a aprender la lógica de la programación) o con la incorporación de diferentes herramientas tecnológicas, como software de programación y/o robótica.

También hemos visto que, en muchos casos, la integración de la robótica se está produciendo de manera extracurricular, cuando es un recurso con muchas potencialidades para ser integrado en el acto didáctico, independientemente del nivel educativo. Resulta interesante analizar cómo los planteamientos tradicionales de la Tecnología 
Educativa retoman su sentido en este punto, porque para que realmente se produzcan cambios significativos es importante un cambio en la estrategia metodológica y tener en cuenta el contexto y las necesidades reales del alumnado.

Esto nos llevaría a replantearnos las experiencias de innovación docente que incorporan la programación o la robótica, para entender que, como hemos aprendido de la historia de la Tecnología Educativa, y al igual que sucede con la docencia, la innovación debe ser contextualizada y estar bien organizada, ya que la mera disposición de la tecnología no supondrá una innovación educativa (Prendes, 2018).

Recordemos que el estudio de los medios es la base de la Tecnología Educativa, medios que definimos como "elementos curriculares, que por sus sistemas simbólicos y estrategias de utilización, propician el desarrollo de habilidades cognitivas en los sujetos, en un contexto determinado, facilitando la intervención mediada sobre la realidad, y la captación y comprensión de la información por el alumno" (Cabero, 1989, p. 60). Esto supone que la robótica educativa representa un recurso más en el aula, con un enorme potencial didáctico, pero que no podemos ubicar como una especialidad en sí misma, provocando un olvido de los supuestos tradicionales de la Tecnología Educativa (Brown, Lewis, y Harcleroad, 1977; Cabero, 2001), como que no existe el supermedio (el medio que nos valga en todas las situaciones educativas), que no hay medios buenos ni malos, y sobretodo, que el aprendizaje no se encuentra en función del medio, sino de la estrategia didáctica que aplique el docente, por lo que el profesor es el elementos más significativo a la hora de integrar la robótica o cualquier otra tecnología en el aula. Olvidar estos supuestos, puede suponer un empobrecimiento pedagógico de la actividad educativa.

Por tanto, cuando valoremos la implementación de estos recursos en el aula, se hace necesario retornar a las bases de la Tecnología Educativa, que partían del análisis de por qué estos medios y por qué ahora, y de la perspectiva de que la integración de la robótica o de cualquier medio en contextos educativos, tiene que ser el resultado de un estudio de muchos componentes contextuales y formativos

\section{- La formación de los futuros docentes:}

Ante este panorama, se hace necesario que los futuros maestros conozcan los aspectos básicos del PC y su relación con la programación y la robótica, así como los recursos disponibles que pueden utilizar en el aula en el marco de estrategias didácticas más completas (Adell et al., 2019; Bocconi et al., 2016; González et al., 2018). Es muy representativo que la mayoría de los profesores que utilizan robótica en las aulas españolas indican que se han formado por su cuenta (MEFP et al., 2018). La rápida evolución de este campo hace que en general, en la mayoría de los países europeos, se promuevan sistemas de formación al profesorado alternativos, como los clubs de programación (INTEF, 2015).

En cuanto a los alumnos, los datos de la Fundación Española para la Ciencia y la Tecnología, en el estudio realizado junto con Google y Everis en 2016, indican que la percepción que los niños tienen respecto a las Ciencias de la Computación es que es una materia demasiado difícil de estudiar y sólo accesible para aquellos que tienen buen expediente académico. Este mismo informe también destaca la importancia de mejorar la formación del profesorado para hacer una buena integración de estos recursos en las aulas.

Ante este panorama, la universidad tiene actuar y empezar a proporcionar la base necesaria para la formación al futuro profesorado en torno a esta temática, partiendo de las bases de la Tecnología Educativa y del conocimiento del PC y su relación con la programación y la robótica, para que la incorporación de estos elementos al currículo no quede de manera anecdótica o se trabaje únicamente de manera extracurricular mediante actividades extraescolares, sino que su entendimiento promueva la incorporación transversal y el desarrollo del espíritu crítico. 


\section{REFERENCIAS}

Adell, J., Esteve, F., Llopis, M. A., y Valdeolivas, M. G. (2017). El Pensamiento Computacional en la formación inicial del profesorado de Infantil y Primaria. En JUTE-2017 (Ed.), XXV fornadas Universitarias de Tecnología Educativa: Aulas y Tecnología Educativa en evolución, Burgos (pp. 151-158). Descargado de http://www3.ubu.es/jute2017/

Adell, J., Llopis-Nebot, M. A., Esteve, F. M., y Valdeolivas, M. G. (2019). El debate sobre el pensamiento computacional en educación. RIED. Revista Iberoamericana de Educación a Distancia, 22, 171-186. https://doi.org/10.5944/ried.22.1.22303

Amiel, T., y Reeves, T. C. (2008). Design-Based Research and Educational Technology: Rethinking Technology and the research agenda. Educational Technology \& Society, 11(4), 29-40.

Balanskat, A., Engelhardt, K., y European Schoolnet. (2015). Computing our future. Computer programming and coding: priorities, school curricula and initiatives across Europe. Descargado de http://www.eun.org/documents/411753/817341/ Computing+our+future_final_2015.pdf/d3780a64-1081-4488-8549-6033200e3c03

Bautista, A., y Alba, C. (1997). ¿Qué es Tecnología Educativa? Autores y significados. Pixelbit. Revista de Medios y Educación, 9-9. Descargado de https://recyt.fecyt.es/index.php/pixel/article/download/61102/37116

Bers, M. U. (2008). Blocks to robots: Learning with technology in the early childhood classroom. New York: Teachers College Press.

Bers, M. U. (2010). The TangibleK Robotics Program: Applied Computational Thinking for Young Children. Early Childhood Research and Practice, 12(2).

Bers, M. U. (2012). Designing digital experiences for positive youth development. New York: Oxford University Press. https://doi.org/10.1093/acprof:oso/9780199757022.001.0001

Bers, M. U. (2018). Codings as a Playground: Programming and Computational Thinking in the Early Childhood Classroom. New York: Routledge. https://doi.org/10.4324/9781315398945

Blikstein, P. (2013). Digital Fabrication and 'Making' in Education: The Democratization of Invention. En C. Büching y J. Walter-Herrmann (Eds.), FabLabs: Of Machines, Makers and Inventors. Transcript Publishers.

Bocconi, S., Chioccariello, A., Dettori, G., Ferrari, A., y Engelhardt, K. (2016). Developing Computational Thinking in Compulsory Education. Descargado de https://publications.jrc.ec.europa.eu/repository/bitstream/JRC104188/ jrc104188_computhinkreport.pdf

Brennan, K., y Resnick, M. (2012). Entrevistas basadas en artefactos para estudiar el desarrollo del Pensamiento Computacional (Pensamiento Computacional) en el diseño de medios interactivos. Vancouver, BC, Canada: American Educational Research Association.

Brown, J. W., Lewis, R. B., y Harcleroad, F. F. (1977). Av Instruction: Technology, media and methods. New YorK: McGrawHill.

Cabero, J. (1989). Tecnología Educativa: utilización didáctica del vídeo. Barcelona: Promociones Y Publicaciones.

Cabero, J. (2001). Tecnología Educativa. Diseño y evaluación de medios aplicados a la enseñanza. Barcelona: Paidos.

Cabero, J. (2003). Replanteando la Tecnología Educativa. Comunicar, 11(21), 23-30. https://doi.org/10.3916/C21-2003-04

Carrera, F. X., y Coiduras, J. L. (2012). Identificación de la competencia digital del profesor universitario: un estudio exploratorio en el ámbito de las ciencias sociales. Revista de Docencia Universitaria, 10(2). https://doi.org/10.4995/redu.2012.6108

Catlin, D., y Woollard, J. (2014). Educational robots and computational thinking. En and others (Ed.), 4th TRtWR \& RIE 2014. International workshop: Teaching robotics \& teaching with robotics. Descargado de http://www.terecop.eu/TRTWR-RIE2014/files/00_WFr1/00_WFr1_18.pdf 
Durán-Cuartero, M., Prendes, M. P., y Gutiérrez-Porlán, I. (2019). Certificación de la Competencia Digital Docente: propuesta para el profesorado universitario. RIED. Revista Iberoamericana de Educación a Distancia, 22(1), 187-205. https://doi.org/10.5944/ried.22.1.22069

Furber, S. (2012). Shut down or restart? The way forward for computing in UK schools. London (UK): The Royal Society.

García, M. A., Deco, C., y Collazos, C. A. (2017). Robotics Based Strategies to Support Computational Thinking: The Case of the Pascual Bravo Industrial Technical Institute. fournal of Computer Science and Technology, 17(01), 59-67. Descargado de http://journal.info.unlp.edu.ar/JCST/article/view/450

García-Valcárcel, A. (2016). Tecnología Educativa: Características y Evolución de una Disciplina. Revista Educación y Pedagogía, 33, 65-87. Descargado de https://www.researchgate.net/publication/277042800_TECNOLOGIA_EDUCATIVA CARACTERISTICAS_Y_EVOLUCION_DE_UNA_DISCIPLINA

Gisbert-Cervera, M., Espuny-Vidal, C., y González-Martínez, J. (2011). INCOTIV. Una herramienta para la autoevaluación diagnóstica de la competencia digital en la universidad. Profesorado, revista de curriculum y formación del profesorado, 15(1), 15-15. Descargado de https://recyt.fecyt.es/index.php/profesorado/article/view/42011/23983

González, J., Estebanell, M., y Peracaula, M. (2018). ¿Robots o programación? El concepto de Pensamiento Computacional y los futuros maestros. EKS. Education in the Knowledge Society, 19, 29-45. https://doi.org/10.14201/eks20181922945

Grover, S., y Pea, R. (2013). Computational thinking in K-12: A Review of the Field. Educational Researcher, 42(1), 38-43. https://doi.org/10.3102/0013189X12463051

INTEF. (2015). Programando nuestro futuro: Programación y codificación Prioridades, currículos e iniciativas en Europa. Descargado de http://code.intef.es/wp-content/uploads/2017/10/ Programando_nuestro_futuro_EUN_INTEF_Octubre_2015.pdf

INTEF. (2017). Marco Común de Competencia Digital Docente. Octubre 2017. Descargado de https://aprende.intef.es/sites/default/files/2018-05/ 2017_1020_Marco-Com\char"00FA|relaxn-de-Competencia-Digital-Docente.pdf

ISTE, y CSTA. (2011). Pensamiento computacional: caja de herramientas para líderes. Descargado de http://eduteka.icesi.edu.co/pdfdir/PensamientoComputacional_Definicion.pdf

Jaramillo, L. M., y Puga, L. A. (2016). El pensamiento lógico-abstracto como sustento para potenciar los procesos cognitivos en la educación. Sophia, Colección de Filosofía de la Educación, 21, 31-55. https://doi.org/10.17163/soph.n21.2016.01

Lye, S. Y., y Koh, J. H. L. (2014). Review on teaching and learning of computational thinking through programming: What is next for K-12? Computers in Human Behavior, 41, 51-61. Recuperado de. https://doi.org/10.1016/j.chb.2014.09.012

Martínez, F. (2016). Sentado en el andén . RIITE. Revista Interuniversitaria de Investigación en Tecnología Educativa(0), 17-22. RIITE. Revista Interuniversitaria de Investigación en Tecnología Educativa. https://doi.org/10.6018/riite/2016/258131

MEFP, INTEF, y CNIIE. (2018). Programación, robótica y pensamiento computacional en el aula. Situación en España y propuesta normativa (MEFP y INTEF, Eds.). Descargado de http://code.intef.es/wp-content/uploads/2018/10/

Ponencia-sobre-Pensamiento-Computacional.-Informe-Final.pdf

Papert, S. (1980). Mindstorms: Children, Computers and Powerful ideas. London: Harvester Press.

Pérez-Narváez, H. O., y Roig-Vila, R. (2015). Entornos de programación no mediados simbólicamente para el desarrollo del pensamiento computacional. Una experiencia en la formación de profesores de Informática de la Universidad Central del Ecuador. REDRevista de Educación a Distancia. Revista de Educación a Distancia, 46, 1-22. https://doi.org/10.6018/red/46/9

Prendes, P. (2004). 2001: una odisea en el ciberespacio. En F. Martínez y P. Prendes (Eds.), 
Nuevas tecnologías y educación (pp. 171-194). Pearson.

Prendes, P. (2018). La Tecnología Educativa en la Pedagogía del siglo XXI: una visión en 3D. RIITE. Revista Interuniversitaria de Investigación en Tecnología Educativa, 4, 6-16.

https://doi.org/10.6018/riite/2018/335131

Reina, M. (2018). Pensamiento Computacional, programación y robótica educativa. Fundamentación y desarrollo curricular. Descargado de http://apprendiendoconrobotica.blogspot.com/2018/09/ pensamiento-computacional-y-robotica.html

Valverde, J., Fernández, M. R., y Garrido, M. C. (2015). El Pensamiento Computacional y las nuevas ecologías del aprendizaje. RED-Revista de Educación a Distancia, 46, 1-18. Descargado de https://www.um.es/ead/red/46/valverde_et_al.pdf

Wing, J. (2006). Computational thinking. CACM Viewpoint. https://doi.org/10.1145/1118178.1118215

Wing, J. (2008). Computational thinking and thinking about computing. Philosophical Transactions Of The Royal Society, 366, 3717-3725.

https://doi.org/10.1098/rsta.2008.0118

Wing, J. (2011). Computational thinking: What and Why. Descargado de https:// www.cs.cmu.edu/link/research-notebook-computational-thinking-what-and-why Zapata-Ros, M. (2015). Pensamiento Computacional: Una nueva alfabetización digital. RED-Revista de Educación a Distancia, 46, 1-47. https://doi.org/10.6018/red/46/4 